\title{
Discussion on Teaching and Exploration of Self-driving Perception Based on SLAM Mode
}

\author{
Han Yajun, Zhang Qingming, Ji Hongyu \\ Jiangsu University of Technology Departments of automotive and traffic engineering, \\ Changzhou Jiangsu, 213001, China
}

\begin{abstract}
With the development of our economy, the progress of society, the improvement of science and technology, the related problems of autopilot have gradually attracted people's attention. With the increasing number of cars, people are not satisfied with the traditional driving mode, from the perspective of future development, self-driving is bound to be able to be applied in the real situation.For the students of related majors, only by understanding the knowledge of autopilot can we lay a solid foundation for the future popularization of autopilot. Based on this, this paper analyzes the teaching and exploration of self-driving perception based on SLAM mode, hoping to beneficial to reality.
\end{abstract}

Keywords: SLAM mode; self-driving; perception teaching

\section{Introduction}

Autopilot is bound to be realized in the future. In fact, autopilot has been studied by relevant people and important research results have been obtained, but it has not been popularized in reality. With the continuous improvement of artificial intelligence technology, all countries have begun to study autopilot, hoping to be invincible in the future fierce competition.If our country wants to be better in the self-driving industry, it needs to pay attention to the teaching of self-driving perception.SLAM is one

https://doi.org/10.47852/bonviewCETR2020010211

This is an open access article published by the BON VIEW PUBLISHING PTE. under the Creative Commons Attributions License. 
of the most important technologies in autopilot, which mainly refers to the construction and positioning of regions and the teaching of autopilot perception based on SLAM mode, which can not only deepen students' impression of autopilot knowledge. At the same time can also promote the future development of the industry.Therefore, the analysis of related problems in this paper has a certain degree of practical significance.

\section{Teaching Value of Self-driving Perception Based on SLAM Mode}

If we want to study the whole problem, we must make clear to some extent the teaching value of self-driving perception based on SLAM mode.It is more important and necessary to carry out selfdriving perception teaching, because the self-driving course itself has. If the teaching can not be carried out reasonably, the development of our country in this field may lag behind other countries. The reason why we should carry out autonomous driving perception teaching on the basis of SLAM mode is mainly because SLAM mode is one of the key technologies in autopilot. Only through SLAM mode can students define the programming language of autopilot and practice it reasonably.However, there are few related people to study the teaching of autopilot perception based on SLAM mode, and there is a certain theoretical blank, but the value of autopilot perception teaching is difficult to deny in reality.

Based on general SLAM, autonomous driving perception teaching can play a key role in the development of students and industries. When students can clearly apply the three-dimensional perception technology in autonomous driving platform, You can better participate in the system development.Therefore, professional teachers in colleges and universities should also strengthen their thinking, deepen their theories, strengthen curriculum construction, take SLAM based LIADR as basic teaching equipment, and take visual camera sensors as auxiliary teaching equipment. The experimental teaching platform of self-driving teaching aids vehicle is built.Only a reasonable construction of a practical teaching platform, can better complete the teaching content. Above, basically is based on the SLAM mode of self-driving perception teaching value.

II. Teaching Measures of Self-driving Perception Based on SLAM Mode

A. Guide students to identify SLAM advantages

To carry out autonomous driving perception teaching based on SLAM mode, students need to make clear the advantages of SLAM.If students can not really understand the advantages of SLAM, it is difficult to really complete the course. There is no doubt that the SLAM is more complex and introduced into China from abroad, so it is difficult for most students to understand it and there are many difficulties in the process of learning. As one of the research hotpots of robot technology, SLAM already exists relevant teaching resources to support teachers to guide and enlighten students. All countries in the world attach great importance to the related technology of robot, among which SLAM have some core properties.SLAM was born abroad as early as 1986. In the process of long-term 
development, the framework of SLAM has been improved and applied in some developed countries. However, the existing research is basically from the point of view of high-precision sensors. Failed to lay a solid foundation for industrialization, naturally can not be used for autopilot.During the past few years, the speed of sensor technology has become more and more important, which makes SLAM pay more and more attention in China. SLAM is a very key technology in autopilot, which involves software, hardware, system application and so on of autopilot. the cost of some sensors has been reduced, which also provides the basis for the realization of industrializationTeachers should guide students step by step, tell students about history related to SLAM, and let students strengthen their understanding of SLAM advantages and importance.

\section{B. Conduct of teaching SLAM framework structures}

SLAM frame structure teaching is the focus of self-driving perception teaching based on SLAM mode. At the same time, it is difficult to carry out SLAM frame structure teaching, there are many details, and a little carelessness may affect the overall teaching effect. Therefore, college teachers must strengthen their thinking, deepen their theory, learn in their spare time, master the frontier knowledge related to SLAM frame structure, collect a lot of teaching resources, enrich curriculum content and reduce students' learning difficulty, To make students understand as the first teaching goal, step by step Into the teaching process.

Teachers should communicate closely with school leaders to build SLAM teaching aids with the goal of SLAM frame structure teaching. In addition, it is necessary to establish visual sensor system and motor control, etc. Complete the capital construction of hardware and software.After designing the software platform, it is necessary to combine the equipment to perfect the system of the course and to explore the fusion method of autopilot and sensor.

SLAM frame structure teaching mainly includes two concrete parts: theory and practice. Both theory and practice are more important and necessary. However, in reality, many teachers only attach importance to theoretical teaching. However, practical teaching is neglected to some extent, which will undoubtedly affect students' learning efficiency.From the point of view of practical teaching, teachers should combine theory teaching and design the fusion algorithm of sensors in the process of building SLAM teaching aids.Besides SLAM in the process of simulating driving in the driveway, once the obstacles can not be identified, the lane line can not be identified, and the obstacles can not be avoided, it is necessary to optimize the computer resources and the motor system reasonably.The data communication of different modules of the system is studied to improve the teaching level of SLAM frame structure and ensure the teaching quality of SLAM frame structure.

C. Define the relationship between modules in detail

A lot of system modules exist in the teaching process of autopilot perception based on SLAM 
mode. Only by making students clear the specific relationship between modules from the point of view of detail can they better accomplish the course objectives.In the process of teaching, college teachers should combine the concrete principle of artificial intelligence, tell students the importance of artificial intelligence in the process of social development, and carry out the teaching of artificial intelligence theory course reasonably.In addition, in order to enable students to learn knowledge that has real application value, teachers also need to strengthen contact with relevant enterprises. The purpose of strengthening contact is to provide students with real learning scenarios, both knowledge base and teaching environment. It should serve the teaching of self-driving perception based on SLAM mode.

Combined with SLAM technology, teachers should explain to students the development process related to autopilot system. In addition, both the construction of system framework and the data processing of sensors need to be fully included.Vehicle dynamics is a very important content, which is closely related to autopilot, but it is not enough to carry out vehicle dynamics teaching only. It also needs the scene design of complete road condition, and puts the SLAM model vehicle into the process of practical exercise.From simple to complex, when students are familiar with the whole process, they can deepen their understanding of autopilot technology.

If there is a certain degree of unknown conditions, then the teacher must guide the students, completely combine the visual sensor with the single-line radar, so as to analyze and study the map in the process of SLAM. Clear its specific features and accurate matching algorithm.In addition, the grid map will be updated continuously in reality, and the updating method of the grid area should be studied, and then the global map and path model should be constructed and the optimization of the global map can be completed by the method and means of manifold.The relationship between each module is more complex. According to the specific requirements of the system, the SLAM and navigation system need to be divided into three different modules, first, sensor information fusion module, second, SLAM module. Finally, the key navigation module is defined, and the adaptation of dynamic environment is considered.

\section{Active feedback on SLAM self-driving teaching}

At the end of teaching, teaching feedback should be carried out, because the basic situation of teaching feedback can show the students' learning results. After the application of SLAM teaching aids, students should complete the study of the whole vehicle construction. Both programming language and perceptual algorithm should be included and integrated into experimental projects.Autopilot courses are aimed at the relevant major students, and the basic knowledge level of the same major students is often different to a certain extent. In order to learn this kind of course well, we should not only have a solid mathematical foundation, but also master the basic knowledge of many programming languages. The multi-sensor radar is a single line, which can only complete the modeling of the conventional scene. 
Once the complex situation is involved, there may be great differences, which will affect the accuracy of the image, so it is necessary to complete the teaching feedback. It is helpful for students to learn and optimize the sensor algorithm.

\section{Conclusion}

To sum up, in recent years, the development of sensor technology is becoming more and more block, which also makes SLAM in China has also been paid attention to by many people. SLAM is a very key technology in autopilot, which involves software, hardware, system application and so on of autopilot. the cost of some sensors has been reduced, which also provides the basis for the realization of industrialization.Based on SLAM mode, autonomous driving perception teaching can not only deepen students' impression of autonomous driving knowledge, but also promote the future development of the industry.To the university teachers, we must strengthen the thought, deepen the theory, strengthen the study in the spare time, constantly master the frontier knowledge related to the SLAM frame structure, and collect a large number of SLAM frame structure teaching resources, enrich the course content, and reduce the students' learning difficultyIn addition, it is necessary to study the update method of grid area, and then construct the global map and path model, and optimize the global map by manifold method and means.

\section{Acknowledgement}

This work is supported in part by Research Fund for the Auto-assisted safe Driving System based on fusion Communication Technology Source: Jiangsu univeristy of Technology Undergraduate Innovation and Entrepreneurship Project No: KYX20027

\section{References}

[1] Focus on artificial intelligence data sovereignty, bionic robot new products are numerous, autopilot carries car manufacturers [J].]; and Automotive Parts ,2020(01):52.

[2] Experimental Teaching Platform for Virtual Simulation of Intelligent Networked Automobile, Chang'an University, J].\& Co., Ltd Experimental Technology and Management ,2019,36(12):19.

[3] Liu Yanbo, Huang Hongcheng, Shi Liangren, Xu Jing. Teaching [J].] of Self-driving Perception Based on SLAM Mode Contemporary Educational Practice and Teaching Research ,2019(21):66-67.

[4] Wang Liansheng, Liu Aiyuan, Wang Zeduo. A Study and Practice on Integrated Capability Assessment System of Aircraft Autopilot Simulator [J].]. The Experimental Technology and Management ,2003(02):38-40 44. 